\title{
Research on the Location Model Based on Clustering Local Search Algorithm under Electronic Commerce
}

\author{
Lei Xu and Jinshuan Peng* \\ Chongqing Key Lab of Traffic System \& Safety in Mountain Cities, Chongqing \\ Jiaotong University, Chongqing 400074, China \\ 37951759@qq.com,pengjinshuan@163.com \\ Corresponding Author: Jinshuan Peng, pengjinshuan@163.com
}

\begin{abstract}
Internet is changing customers' consumption patterns and the manufactures' sale model. With the development of the computer network technology and the electronic commerce, more and more firms establish the electronic sale channel and get great profits. The huge supply chain network is established through the new idea and the technology. In this paper, we propose an improved local search algorithm- clustering local search algorithm (CLSA) to solve the hybrid location problem. We apply this method to distribution center location model and get the optimal solution. Result shows that this method can avoid the exponential explosion and get a good solution. In numerical analysis, we compare this method with simulated annealing method and ant searching algorithm. The numerical analysis demonstrates that this CLSA method not only classifies simply and flexibly, but also has the characteristic of fast search speed and small search space.
\end{abstract}

Keywords: E-commerce, CLSA, Hybrid location problem

\section{Introduction}

Location problem belongs to combinational optimization problem. With in-depth study of this problem, they produce many models and algorithms. These problems are NP-hard problem $[1,2]$. K lose A and Drexl A proposed the transformation models in different conditions according to continuous model, internet model and mixed integer programming model [3]. They analyzed the mixed integer programming model and proposed the mixed integer programming models in 7 conditions. At the same time, they reviewed the different models and the algorithms. Aikens $\mathrm{C} \mathrm{H}$ proposed 9 location models including simple location model, location model of capacity constraints, the location model of demand changes and the dynamic location model [4]. The objective function is to minimize the transportation cost and location cost. Revelle C S and Eiselt $\mathrm{H}$ A thought that each formulation had differences and similarities relative to the others, but the peculiarities of each problem provided the fuel for the hundreds of investigations [5].

Joel S.E. Teo, Eiichi Taniguchi and Ali Gul Quresh proposed a logistics measure for an urban road network in an e-commerce delivery system environment. Most notable contribution of this evaluation methodology is the combination of vehicle routing and scheduling problem with time window (VRPTW), auction theory and reinforcement learning in a multi-agent framework [6]. The results of the model show that Governmentdriven City Logistics measures has the potential of reducing truck emission when the administrator learns and prices the road links. Using data from online customer ratings, we explore how the relationships between logistics performance and customer loyalty are affected by risk characteristics of products and efficiencies of the websites. Ramakrishnan explored how the relationships between logistics performance and customer loyalty are affected by risk characteristics of products and efficiencies of the websites with the data 
from online customer ratings [7]. Wang Ying and Sang Dayong analyzed the relationships between the 3PL and supply chain members [8]. The authors suggested that only when the 3PL reengineers its logistics business process to accommodate the customer could it maximize the value of the customer. Markus Hesse thought the significance of electronic commerce (e-commerce) for freight transport, logistics and physical distribution, regarding both business to business and business to consumer commerce.

Distribution problem can be divided into the three main research directions. One is the model and algorithm of the distribution center location model. Other one is the model and algorithm of the distribution path optimization problems. The last one is the model and algorithm of locating the vehicle routing problems. Among them, the typical research method of the continuous model uses the gravity method to solve the location problem of Euclidean distance which is proposed by Xingyuan Zeng [9]. The research method of the discrete classical model is Kuehn-Hamburger model [10], Baumyr Wolf method [11], the mixed integer programming method [12], Capacitated Facility Location method [13] and P-middle value problems [4]. The dynamic location model is to solve the facility location problem. The model makes the total long-term cost minimum [14-15]. The stochastic model and its research methods are mainly divided into two categories. They are probability method and the scenario planning method. The system input parameters of these two methods are uncertain [16]. Aiming at different location problems and models, the problems we discussed are more and more complex. A heuristic algorithm is the solving direction.

Heuristic algorithm is proposed by Gillett et al which is from solving TSP (traveling salesman problem) [17]. Firstly, heuristic algorithm computes the polar coordinates of the points we will visit and rank the points according to the sizes of the angles. Secondly, heuristic algorithm optimizes the sub paths according to TSP optimization algorithm. Zhong Y and Cole M H present a guided local search heuristic to solve a vehicle routing problem with backhauls and time windows [18]. Customer precedence requires that all linehaul customers be visited before any backhaul customer. The basic approach is to construct an initial infeasible solution and then use a guided local search to improve the solution feasibility and quality. Numerical analysis shows that the new heuristic can solve problems better than the best solutions that have appeared in the literatures.

In this paper, we will solve a hybrid location problem. There is no clear alternative distribution center and all customer points can be as alternative distribution centers. In this alternative distribution center, using a simple heuristic algorithm can not avoid the exponential explosion. Therefore, we propose a CLSA method. In this method, we use clustering method to construct the initial solution to simplify the scale of the problem. Then, we use local search algorithm to get the optimal solution. The structure of this paper is as follows. The first part is the introduction. The second part is the correlated conditions to establish the location model. The third part is improved local search algorithm CLSA. The fourth part is the numerical analysis and the last part is the conclusion.

\section{The Correlated Conditions to Establish the Location Model}

\subsection{Assumptions}

(1) The candidate locations of the logistics enterprise are sure. (2) Each customer demand point can be established as the distribution center. We define the reconstruction cost as zero. That means, no matter which customer point we choose, the reconstruction cost is basically the same and the comparison cost is zero. (3) We assume that some customers demand points are designated as the distribution centers and the other customer demands are distributed by these distribution centers. (4) Goods which belong to bulk buying clients are delivered to the customers directly without the distribution center. (5) 
The freight is a linear function of the distance. (6) Except the transportation costs, the other cost are as the fixed costs. (7) Each customer is served by the fixed distribution center and the demand is fixed. (8) The ability of the logistics enterprise and the distribution center can satisfy the demand.

\subsection{The Parameters of the Model}

The specific parameters are as follows. $N\left\{n_{i} \mid i=1,2, \cdots, N\right\}$ is a serious of the logistics enterprise node set in $N . K\left\{k_{i} \mid i=1,2, \cdots, K\right\}$ is a serious of the customer node set in $K$. $C_{i k}$ is the unit transportation cost from the logistics enterprise $i$ to the costumer $k . C_{j k}$ is the unit transportation cost from the customer $j$ to the customer $k(j \neq k) . W_{i k}$ is the transportation volume from the logistics enterprise $i$ to the costumer $k . f_{i}$ is the fixed cost for the operation of the logistics enterprise $i . Q_{k}$ is the quantity demand of the customer $k$.

\subsection{The Decision Variables}

The specific formulation is as follows.

If the logistics enterprise is enabled, $Y_{i}=1$. Otherwise, $Y_{j}=0$.

If the customer $j$ is selected as the distribution center, $Y_{j}=1$. The condition is $j \in K$. Otherwise, $Y_{j}=0$.

If the customer $k$ is distributed by the customer $j, Y_{j k}=1$. The conditions are $j, k \in K$ and $j \neq k$. Otherwise, $Y_{j k}=0$.

If the customer $j$ is distributed directly by the logistics enterprise $i, Y_{i k}=1$. The condition are $i \in N$ and $k \in K$. Otherwise, $Y_{i k}=0$.

\subsection{The Location Model}

$$
\min z=\sum_{i \in N} \sum_{k \in K} C_{i k} Q_{k} d_{i k} Y_{i k}+\sum_{j \in K} \sum_{k \in K} C_{j k} Q_{k} d_{j k} Y_{j k}+\sum_{i \in N} f_{i} Y_{i}
$$

The constraint conditions are as follows.

$$
\begin{gathered}
\sum_{i \in N} Y_{i}=1 \\
\sum_{j \in K} Y_{j k}=1, k \in K \\
\sum_{k \in K} Q_{k} Y_{j k} \leq w, j \in K \\
Y_{j k} \leq Y_{j}, j \in K, k \in K \\
Y_{i k} \leq Y_{i}, i \in N
\end{gathered}
$$

In the formula (1), the objective function is the minimum of the fixed cost. The fixed cost contains the transportation cost from the logistics enterprise to the distribution center, the transportation cost from the distribution center to the customer and the fixed cost of the logistics enterprise. The constraint condition (2) ensures to enable one logistics enterprise. The constraint condition (3) ensures that there is only one distribution center for the service in the customer point. The constraint condition (4) ensures the customer delivery quantity of the distribution center does not exceed the maximum vehicle load. The constraint conditions (5) and (6) ensure that if there is no logistics enterprise of distribution point location, no distribution point or the customer correspondence is here. 


\section{The Improved Local Search Algorithm CLSA (Clustering Local Search Algorithm)}

\subsection{The Solution Description of the Improved Local Search Algorithm CLSA}

In this paper, we use the improved local search algorithm CLSA to solve this model. In the first step, we adopt the density weighted fuzzy $c$ means clustering method to classify all the customer points. In the second step, we group and adjust the cluster results by using the scanning method. Then, we use the traditional iterative method to obtain the best possible solution. The third step is to optimize the initial solution by the 1-mobile and the 1-exchange.

Because generating a clustering center is equivalent to produce a distribution center at least, the number of the cluster centers has an important influence on the solution. In order to reduce the number of the distribution center, we set that $c \leq \sum_{k \in K} Q_{k} / W$. Among them, $k$ represents the total number of the customers. $Q_{k}$ is the distribution volume of the $k t h$ customer. $W$ is the load of the vehicle.

\subsection{The Density Weighted Fuzzy $c$ Means Clustering Method}

We know the categories $2 \leq c \leq n, \tilde{U} \in M_{f c}$ and assume $v_{i}=\sum_{k=1}^{n} u_{i k} x_{k} / \sum_{k=1}^{n} u_{i k}, \forall i$. We suppose $v_{i}$ as the fuzzy $c$ clustering center of the category $i$. The fuzzy object function of the mean clustering model is as follows.

$$
\begin{gathered}
\min z(\tilde{u}, V)=\sum_{i=1}^{c} \sum_{k=1}^{n}\left(u_{i k}\right)^{m}\left\|x_{k}-v_{i}\right\|^{2} \\
v_{i}=\frac{\sum_{k=1}^{n}\left(u_{i k}\right)^{m} x_{k}}{\sum_{k=1}^{n}\left(u_{i k}\right)^{m}}, \forall i \\
\tilde{U} \in M_{f c}
\end{gathered}
$$

Among them, $m \geq 1$ is a given number. $\tilde{U}=\left[u_{i k}\right]$ is the fuzzy classification $\operatorname{matrix}\left(u_{i k} \in[0,1], \forall i, k\right) . \forall i$ is the clustering center of the category $i$.

The constraint condition of the objective function (7) is $\sum_{i=1}^{c} u_{i k}=1$. And we introduce the Lagrange multipliers $\lambda$.

$$
\begin{gathered}
(\tilde{u} ; V)=\sum_{i=1}^{c} \sum_{k=1}^{n} D_{k}\left(u_{i k}\right)^{m}\left\|x_{k}-v_{i}\right\|_{G}^{2}+\sum_{k=1}^{n} \lambda\left(1-\sum_{i=1}^{c} u_{i k}\right) \\
D_{k}=\sum_{y=1}^{n} \exp \left(-\frac{h \cdot\left\|x_{k}-x_{y}\right\|_{1}}{S T D}\right)
\end{gathered}
$$

$D_{k}$ is the potential energy function, and $h$ is the Resolution factor, STD is the standard deviation of the input data.

Seeking the partial derivative of $\lambda, u_{i k}$ and $v_{i}$, we can get the necessary conditions of the local optimal.

$$
v_{i}=\frac{\sum_{k=1}^{n} D_{k}\left(u_{i k}\right)^{m} x_{k}}{\sum_{k=1}^{n} D_{k}\left(u_{i k}\right)^{m}}, i=1,2, \cdots, c
$$


The algorithm steps are as follows.

$$
u_{i k}=\frac{\left(\frac{1}{\left\|x_{k}-v_{i}\right\|_{G}^{2}}\right) \frac{1}{m-1}}{\sum_{j=1}^{c}\left(\frac{1}{\left\|x_{k}-v_{i}\right\|_{G}^{2}}\right) \frac{1}{m-1}}, i=1,2, \cdots, c
$$

The step 1 is to select $c(2 \leq c \leq n), m(1<m<\propto)$ and the matrix $G$. Initializing $\tilde{U} \in M_{f c}$. Then we assume the iteration index $L=0$.

The step 2 is to calculate the density weighted $c$ fuzzy clustering center $v_{i}^{[L]}$ according to the formula (12).

The step 3 is to calculate the new membership matrix $\tilde{U}^{(L+1)}$ by $v_{i}^{[L]}$ when $x_{k}=v_{i}^{[L]}$ according to the formula (13). Otherwise, $. u_{i k}=\left\{\begin{array}{l}1 \ldots \ldots j=i \\ 0 \ldots \ldots j \neq i\end{array}\right.$

The step 4 is to calculate the stopping criterion $\Delta=\left\|\tilde{U}^{(L+1)}-\tilde{U}^{(L)}\right\|_{G}$. If $\Delta>\varepsilon, L=L+1$ goes to the step 2. If $\Delta \leq \varepsilon$, end the calculation.

The step 5 is to determine the exact category by using the maximum membership degree principle. That is, if one $i_{0}$ satisfies the condition $u_{i_{0} k}=\max _{\forall i}\left\{u_{i k}\right\}, i_{0}$ is the ownership category of the $x_{k}$.

\subsection{Constructing the Initial Solution Based on the Scanning and the Iterative Method}

Firstly, we use the scanning method to group and adjust the cluster center. Secondly, we adopt the traditional iterative method to get the best possible initial solution. At last, we make the results in the record. Cycling the process, we compare these results and find out the satisfactory computational results as the initial solution.

The step 1 is to select a group from the above clusters.

The step 2 is to take a customer as the origin of the polar coordinates. We define a connected line of one customer and an origin as the zero angle and establish the polar coordinates.

The step 3 is to transform the coordinate system of the position for the customers that have been divided. All the coordinate systems are transformed into the polar coordinate systems.

The step 4 is to establish a group starting from the minimum angle of the customer. According to the counterclockwise, the customers are added to the group one by one until the constraints of the customer beyond the constraint condition.

The step 5 is to establish a new group if the customer constraints exceeded the constraint condition. Then, we add the customers to the group according to the counterclockwise.

The step 6 is to repeat the step 4 until all of the customers are grouped.

The step 7 is to calculate the cost lowest coordinate $(x, y)$ of each customer point in each group by using the iterative. If we want $F(x, y)$ minimum, we must make $\frac{\partial F(x, y)}{\partial x}=0$ and $\frac{\partial F(x, y)}{\partial y}=0$.

The step 8 is to calculate the customer point which is nearest the $(x, y)$ in the group. Then, the customer point is the distribution point in this group.

The step 9 is to recalculate $F(x, y)$ according to the selected distribution point. Then the results are recorded in the record table.

The step 10 is to choose a new location as the pole. Then, we use the scanning method to group and transform to the step 2. 
The step 11 is to repeat the steps 2-10 until finding out the satisfied results as the initial solution.

\subsection{The Local Search Algorithm}

We adopt the local search algorithm to optimize the initial solution. The specific process is as follows.

(1) 1 -move

1-move is the heuristic algorithm which is the same to the operative symbols $(1,0)$ and $(0,1)$. It can improve the quality of the solution and the feasibility of the poor solution effectively. The specific operation method is as follows. We delete a customer point from a group. And then we insert the customer point into another group.

The neighborhood structure is as follows. We make the distribution point as the origin in each group. And we use the farthest customer distance as the radius of the circle. The customer point in this circle is as the neighborhood of each distribution point.

(2)1-exchange

The main ideal of the 1-exchange method is to delete the two customers from two groups, then insert them into each other's group. The method can effectively increase the ability of the local search. The neighborhood structure is the same to the 1-move. The neighborhood radius may be slightly larger.

(3)Determine the location of the distribution point and the logistics base.

\section{Numerical Analysis}

We assume that there are two candidate locations $B_{1}$ and $B_{2}$. The position coordinates are $B_{1}(0,0), B_{2}(52,48)$ and $B_{3}(79,93)$. The transportation expense is $1 . f_{1}=80, f_{2}=100$. The biggest cargo capacity is 4 tons. I, II and III are bulk buying clients. 1-35 are General customer points. The basic information of the customer are shown in Table.1

Table 1. The Basic Information

\begin{tabular}{|c|c|c|c|}
\hline \multirow{2}{*}{ Customer point } & \multicolumn{2}{|c|}{ coordinate } & \multirow{2}{*}{ cargo capacity } \\
\hline & ordinate & ordinate & \\
\hline $\mathrm{I}$ & 46 & 15 & 4 \\
\hline II & 52 & 67 & 4 \\
\hline III & 3 & 48 & 4 \\
\hline 1 & 36 & 1 & 0.4 \\
\hline 2 & 27 & 4 & 1.3 \\
\hline 3 & 42 & 7 & 0.7 \\
\hline 4 & 25 & 4 & 1.9 \\
\hline 5 & 11 & 6 & 1.6 \\
\hline 6 & 43 & 8 & 0.8 \\
\hline 7 & 8 & 5 & 0.6 \\
\hline 8 & 39 & 9 & 1.2 \\
\hline 9 & 26 & 68 & 1.0 \\
\hline 10 & 57 & 32 & 0.5 \\
\hline 11 & 6 & 59 & 2.1 \\
\hline 12 & 21 & 48 & 1.7 \\
\hline 13 & 48 & 21 & 0.5 \\
\hline 14 & 28 & 17 & 0.3 \\
\hline 15 & 31 & 40 & 1.9 \\
\hline 16 & 47 & 28 & 0.6 \\
\hline 17 & 55 & 9 & 0.4 \\
\hline 18 & 35 & 16 & 1.3 \\
\hline 19 & 20 & 21 & 1.1 \\
\hline
\end{tabular}




\begin{tabular}{|c|c|c|c|}
\hline 20 & 45 & 8 & 0.3 \\
\hline 21 & 7 & 29 & 0.4 \\
\hline 22 & 34 & 13 & 0.2 \\
\hline 23 & 16 & 39 & 1.0 \\
\hline 24 & 40 & 14 & 0.8 \\
\hline 25 & 32 & 17 & 1.2 \\
\hline 26 & 14 & 38 & 0.4 \\
\hline 27 & 41 & 44 & 0.8 \\
\hline 28 & 20 & 27 & 1.3 \\
\hline 29 & 35 & 49 & 0.9 \\
\hline 30 & 8 & 18 & 0.4 \\
\hline 31 & 3 & 4 & 0.9 \\
\hline 32 & 2 & 22 & 1.5 \\
\hline 33 & 7 & 15 & 0.6 \\
\hline 34 & 15 & 35 & 1.1 \\
\hline 35 & 26 & 17 & 0.6 \\
\hline
\end{tabular}

\subsection{Set Partitioning}

Firstly, we confirm the number of categories $c=[32.5 / 5]+1=7$. Then, we use the density weighted $\mathrm{C}$-means clustering method to divide the customer points into 7 categories category. The first is $1,2,3,6,20$; the second category is $4,10,13,14,16,18,25,35$; the third category is $15,19,21,29$; the fourth category is $5,7,31,33$; the fifth category is $8,17,22,24,27$; the sixth category is $9,11,32,34$; and the seventh category is $12,23,28,26,30$. At last, we set $B_{1}(0,0)$ as the pole and establish a polar coordinates.

\subsection{Reclassification Adjustment}

Then we adjust the reclassification. The steps are as follows.

Step 1 is to examine the classifications. The second category does not conform to the constraints. Therefore, we scan the decomposition.

Step 2 is to re-scan the second category. After removing the customer point 14, the fifth category satisfies constraint conditions.

Step 3 is to add the point 14 into the fifth category.

Step 4 is to examine the classifications. The fifth category does not conform to the constraints. Therefore, we scan the decomposition.

Step 5 is to re-scan the fifth category. After removing the customer point 27, the fifth category satisfies constraint conditions.

Step 6 is to add the point 27 into the sixth category.

Step 7 is to re-scan the sixth category. After removing the customer point 34 , the sixth category satisfies constraint conditions.

Step 8 is to add the point 34 into the seventh category.

Step 9 is to examine the classifications again. All categories satisfy constraint conditions.

The final classification results are as shown.

The first category is $1,2,3,6,20$; the second category is $4,10,13,16,18,25,35$; the third category is $15,19,21,29$; the fourth category is $5,7,31,33$; the fifth category is $8,14,17,22,24$, ; the sixth category is $9,11,27,32$; and the seventh category is $12,23,28,26,30,34$.

Then we use iteration method to confirm the coordinates of distribution centers and the initial distribution plan. We use customer points $3,16,19,31,17,9,23$ as the distribution center though logistics base $B_{1}(0,0)$. The total cost is 2205.40 .The results are shown as Table. 2 
Table 2. Initial Distribution Schemes

\begin{tabular}{|c|c|c|}
\hline Category & Distribution center & The shipping quantity \\
\hline $1,2,3,6,20$ & 3 & 3.5 \\
\hline $4,10,13,16,18,25,35$ & 16 & 6.6 \\
\hline $15,19,21,29$ & 19 & 4.3 \\
\hline $5,7,31,33$ & 31 & 3.7 \\
\hline $8,14,17,22,24$ & 17 & 2.9 \\
\hline $9,11,27,32$ & 9 & 5.7 \\
\hline $12,23,28,26,30,34$ & 23 & 5.8 \\
\hline Cost & \multicolumn{2}{|c|}{1258.68} \\
\hline $\begin{array}{l}\text { The bulk buying } \\
\text { clients cost }\end{array}$ & \multicolumn{2}{|c|}{846.72} \\
\hline $\begin{array}{c}\text { Logistics base fixed } \\
\text { fee }\end{array}$ & \multicolumn{2}{|c|}{100} \\
\hline Total cost & \multicolumn{2}{|c|}{2205.40} \\
\hline
\end{tabular}

Then, we use local search algorithm to optimize the initial solution. We use customer points $2,16,19,7,17,27,23$ as the distribution center though logistics base $B_{1}(0,0)$. The total cost is 2126.13 .The results are shown as Table. 3

The final optimized initial distribution schemes is shown as Table. 3

Table 3. Final Optimized Initial Distribution Schemes

\begin{tabular}{|c|c|c|}
\hline Category & Distribution center & The shipping quantity \\
\hline $1,2,3,6,20$ & 2 & 3.5 \\
\hline $4,10,13,16,18,25,35$ & 16 & 6.6 \\
\hline $15,19,21,29$ & 19 & 4.3 \\
\hline $5,7,31,33$ & 7 & 3.7 \\
\hline $8,14,17,22,24$ & 17 & 2.9 \\
\hline $9,11,27,32$ & 27 & 5.7 \\
\hline $12,23,28,26,30,34$ & 23 & 5.8 \\
\hline Cost & \multicolumn{2}{|c|}{846.72} \\
\hline The bulk buying clients cost & \multicolumn{2}{|c|}{100} \\
\hline Logistics base fixed fee & 2126,13 \\
\hline Total cost & \multicolumn{2}{|c|}{} \\
\hline
\end{tabular}

We use local search algorithm to optimize the initial solution and improve the initial solution. The quality of the solution improves $3.72 \%$.

\subsection{Comparison of Other Algorithms (Simulated Annealing Method, Ant Algorithm)}

We use simulated annealing method and ant searching algorithm to get the solution result. When we use simulated annealing method, we choose customer points $3,13,21,31,14,23,27$ as the distribution center though logistics base $B_{1}(0,0)$. When we use ant searching algorithm, we choose customer points $3,13,21,7,14,23,28$ as the distribution center though logistics base $B_{1}(0,0)$. The total cost is 2126.13 .The results are shown as Table. 4 and Table.5

Table 4. Solution Result of Simulated Annealing Method

\begin{tabular}{|c|c|c|c|}
\hline \multirow{2}{*}{$\begin{array}{c}\text { Simulated } \\
\text { annealing }\end{array}$} & Category & Distribution center & The shipping quantity \\
\cline { 2 - 4 } & $1,2,3,4,20$ & 3 & 3.5 \\
\hline
\end{tabular}




\begin{tabular}{|c|c|c|c|}
\hline \multirow[t]{10}{*}{ method } & $6,10,13,16,18$ & 13 & 6.6 \\
\hline & $15,19,21,25,29$ & 21 & 4.3 \\
\hline & $5,7,31,33,35$ & 31 & 3.7 \\
\hline & $8,14,17,22,24$ & 14 & 2.9 \\
\hline & $9,11,23,32,34$ & 23 & 5.7 \\
\hline & $12,, 28,26,27,30$ & 27 & 5.8 \\
\hline & Cost & \multicolumn{2}{|c|}{1257.09} \\
\hline & Bulk buying clients cost & \multicolumn{2}{|c|}{846.72} \\
\hline & Logistics base fixed fee & \multicolumn{2}{|c|}{100} \\
\hline & Total cost & \multicolumn{2}{|c|}{2203.81} \\
\hline
\end{tabular}

Table 5. Solution Result of Ant Searching Algorithm

\begin{tabular}{|c|c|c|c|}
\hline \multirow{12}{*}{$\begin{array}{c}\text { Ant } \\
\text { searching } \\
\text { algorithm }\end{array}$} & Category & Distribution center & The shipping quantity \\
\hline & $1,2,3,4,20$ & 3 & 3.5 \\
\hline & $6,10,13,16,18$ & 13 & 6.6 \\
\hline & $19,21,25,29$ & 21 & 4.3 \\
\hline & $5,7,31,33,35$ & 7 & 3.7 \\
\hline & $8,14,15,17,22,24$ & 14 & 2.9 \\
\hline & $9,11,23,32,34$ & 23 & 5.7 \\
\hline & $12,, 28,26,27,30$ & 28 & 5.8 \\
\hline & Cost & \multicolumn{2}{|c|}{1428.92} \\
\hline & Bulk buying clients cost & \multicolumn{2}{|c|}{846.72} \\
\hline & Logistics base fixed fee & \multicolumn{2}{|c|}{100} \\
\hline & Total cost & \multicolumn{2}{|c|}{2375.64} \\
\hline
\end{tabular}

Table 6. Comparison of Different Algorithms

\begin{tabular}{|c|c|c|c|}
\hline Algorithm & Distribution center & Cost & Base \\
\hline Simulated annealing method & $3,13,21,31,14,23,27$ & 2203.81 & $B_{1}(0,0)$ \\
\hline Ant searching algorithm & $3,13,21,7,14,23,28$ & 2375.64 & $B_{1}(0,0)$ \\
\hline CLSA & $2,16,19,7,17,32,23$ & 2126,13 & $B_{1}(0,0)$ \\
\hline
\end{tabular}

Form the contrast experiment, we can see that the solution of CLSA method is better than simulated annealing method and ant searching algorithm. This means that CLSA method is more feasible and excellent.

\section{Conclusion}

With the rapid development of Internet and the information changing, the e-commerce makes an immense impact on the economic life. However, the laggard logistics in our country restricts seriously the development of the electronic commerce. In this paper, we research the location problem and complete the following works. (1) Firstly, we propose the CLSA (clustering local search algorithm). (2) Secondly, we apply this method to distribution center location model and get the optimal solution. (3) At last, we compare this method with simulated annealing method and ant searching algorithm. The numerical analysis shows that this CLSA method not only classifies simply and flexibly, but also has the characteristic of fast search speed and small search space. We can get good results with CLSA. 


\section{Acknowledgments}

This work is supported by Natural Science Foundation of Chongqing (cstc2013jcyjA30015) and Special Research Plan of Education Department of Shaanxi Provincial Government (2013JK0219).

\section{References}

[1] G. CornujoisG, M. L. Fisher and G. L. Nemheuser, "Location of bank accounts to optimize float: a analytic study of exact and approximate algorithms," Management Science, vol. 23 no. 8, (1977), pp. 45-56.

[2] B. Goldengorin, D. Ghosh and G. Sierksma, "Branch and peg algorithms for the simple plant location problem," Computers \& Operations Research, vol. 34 no. 2, (2004), pp. 241-255.

[3] A. Klose and A. Drexl, "Facility location models for distribution system design," European Journal of Operational Research, vol. 162, (2005), pp. 4-29.

[4] C. H. Aikens, "Facility location models for distribution planning," European Journal of Operational Research, vol. 22 no. 2, (1985), pp. 1-19.

[5] C. S. Revelle and H. A. Eiselt, "Location analysis: a synthesis and survey," European Journal of Operational Research, vol. 162, (2005), pp. 423-447.

[6] J. S. E. Teo, E. Taniguchi and A. G. Quresh, "Evaluating city logistics measure in e-commerce with multi-agent systems," Procedia - Social and Behavioral Sciences, vol. 39, (2012), pp. 349-359.

[7] R. Ramanathan, "The moderating roles of risk and efficiency on the relationship between logistics performance and customer loyalty in e-commerce", Transportation Research Part E: Logistics and Transportation Review, vol. 46 no. 6, (2010), pp. 950-962.

[8] Y. Wang and D. Y. Sang, "Multi-agent framework for third party logistics in E-commerce," Expert Systems with Applications, vol. 29 no. 2, (2005), pp. 431-436.

[9] X. Y. Zeng, "Distribution of eigenvalues of large Euclidean matrices generated from ellipsoid," Statistics \& Probability Letters, vol. 91, (2014), pp. 181-191.

[10] J. S. Kim and D. H. Lee, "A restricted dynamic model for refuse collection network design in reverse logistics," Computers \& Industrial Engineering, vol. 66 no. 4, (2014), pp. 1131-1137.

[11] D. Y. Jiang, "Study on Location Selection based on Multi-Node Logistics," Railway Transport and Economy, vol. 27 no. 8, (2005), pp. 24-26.

[12] K. Zhou, X. Chen and Z. J. Shao, "Heterogeneous parallel method for mixed integer nonlinear programming," Computers \& Chemical Engineering, vol. 66, (2013), pp. 290-300.

[13] T. Küçükdeniz, A. Baray, K. Ecerkale and Ş. Esnaf, "Integrated use of fuzzy c-means and convex programming for capacitated multi-facility location problem," Expert Systems with Applications, vol. 39 no. 4, (2012), pp. 577-580.

[14] P. N. Thanh, N. Bostel and O. Péton, "A dynamic model for facility location in the design of complex supply chains," International Journal of Production Economics, vol. 113 no. 2, (2008), pp. 678-693.

[15] E. Gebennini, R. Gamberini and R. Manzini, "An integrated production-distribution model for the dynamic location and allocation problem with safety stock optimization," International Journal of Production Economics, vol. 112 no. 1, (2009), pp. 286-304.

[16] S. L. Wu, J. J. Chen and W. C. Chou, "Cell-related location area planning for 4G PCS networks with variable-order Markov model Original," Journal of Systems and Software, vol. 86 no. 10, (2013), pp. 2688-2699.

[17] B. E. Gillett and L. R. Miller, "A heuristic algorithm for the vehicle dispatch problem," Operations Research, vol. 22 no. 2, (1974), pp. 340-349.

[18] Y. J. Zhong and M. H. Cole, "A vehicle routing problem with backhauls and time windows: a guided local search solution," Transportation Research Part E: Logistics and Transportation Review, vol. 41 no. 2, (2005), pp. 131-144

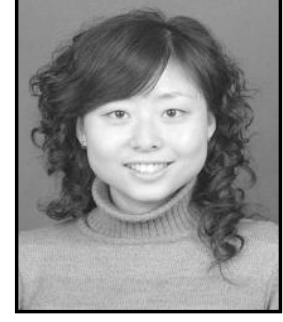

\section{Authors}

Lei Xu, Female, Doctor of Logistic Engineering, Associate Professor. She received her Ph.D. in logistic engineering (2011) from Chang'an university. Now she is an assistant professor of traffic management, Chongqing Jiaotong University. Her current research interests include E-commerce implementation evaluation and supply chain emergency management. 


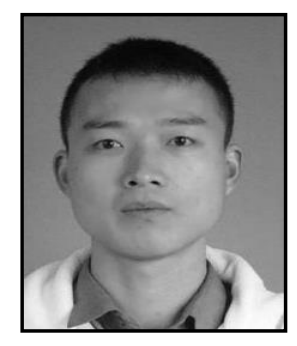

Jinshuan Peng, Male, Doctor of Engineering, Associate Professor. He received his Ph.D. in vehicle operation engineering (2012) from Chang'an university. Now he is an assistant professor of transportation, Chongqing Jiaotong University. His current research interests include driving behavior and E-commerce implementation evaluation. 
International Journal of Multimedia and Ubiquitous Engineering

Vol.10, No.6 (2015) 